\title{
Performance Analysis of Cloud under different Virtual Machine Capacity
}

\author{
V.Suresh Kumar \\ Research Scholar \\ M.S University \\ Tirunelveli
}

\author{
M.Aramudhan, PhD. \\ Associate Professor \\ PKIET
}

\begin{abstract}
Cloud computing is an upcoming, novel, realistic paradigm to host and deliver internet services. Cloud computing helps to plan accurately for provisioning of hardware or software and also eradicates the need of high-priced computing hardware and maintenance. Using virtualization, clouds handle shared collection of physical resources in a huge user base having differing requirements. Hence, clouds are substitute for grids, clusters and supercomputers, but in challenging scientific computing workloads virtualization leads to performance penalties. This paper investigates cloud performance analysis under different Virtual Machine (VM) capacity. VM parameters like RAM and processors number are varied, and resource allocation, CloudSim debt and VM percentage used are also studied.
\end{abstract}

\section{General Terms}

Computing, Performance.

\section{Keywords}

Cloud Computing, Virtualization, Virtual Machines, Cloudsim Debt

\section{INTRODUCTION}

Cloud Computing refers to applications delivered as services on the Internet and data centres hardware/systems software which provides such services. Datacenter hardware/software is a Cloud. Clouds [1] attempt to power next generation datacenters by combining them as a virtual services network (hardware, database, user-interface, application logic) to enable users to access and deploy applications on demand from anywhere in the world at competitive costs based on users QoS (Quality of Service) requirements [2].

Cloud environment's computing power is supplied through many data centers installed in hundreds to thousands of servers [3]. Cloud based data centres' layered architecture is shown in Figure 1 [4]. Massive physical resources (storage servers and application servers) that power data centers are at the lowest layer and are transparently managed by higher level virtualization [5] services and toolkits allowing capacity sharing among virtual servers' instances. The latter are isolated from each other helping achieve fault tolerant behaviour in an isolated security context.

Datacenter provides core infrastructure level services (hardware, software) offered by a Cloud computing environment's resource providers encapsulating a set of computer hosts (blade servers) which are either homogeneous or heterogeneous regarding resource configurations (memory, cores, capacity, and storage). Also, datacenter component initiates a generalized resource provisioning component implementing policies to allocate bandwidth, memory, and storage devices.

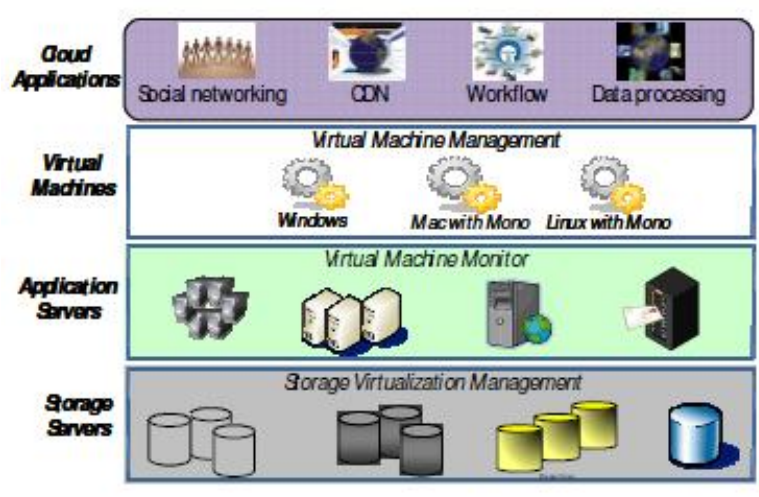

Figure 1: Typical Cloud-based Data Center

Virtual Machine (VM) whose life cycle management is the responsibility of Host component can simultaneously instantiate multiple VMs and allocate cores based on predefined processor sharing policies (space-shared, timeshared). Every VM component can access a component storing VM related characteristics like memory, processor, storage, and VM's internal scheduling policy extended from an abstract component called VM Scheduling.

Cloud computing has many key advantages: Lowers entry cost dramatically for small firms aiming to benefit from computer-intensive business analytics. Provides almost immediate access to hardware resources with no upfront capital investments for users, thus, resulting in quicker marketing in many businesses. Cloud computing can lower IT innovation barriers as seen from many promising start ups, from ubiquitous online applications like Facebook and Youtube. Cloud computing enables easy service for upscaling of enterprises based on client demand. Cloud computing ensures new applications classes and delivers services like mobile interactive applications which are context-aware, environment, and location, parallel batch processing [6].

Three core cloud computing technologies are - virtualization, multi-tenancy and Web services. Virtualization hides a computing platform's physical characteristics from users and instead presents an abstract, emulated computing platform [7] which behaves like an independent system, but unlike it can be configured on demand, maintained and replicated easily. Computing infrastructure is thus better utilized, leading to reduced upfront/operational costs. While the virtualization is prevalent from the 1960 s, only recently have the computing power and networking resources caught up to deliver seamless performance in an emulated system that users are used to on personal computers. Multi tenancy is a related concept whereby a single application software instance benefits many clients ensuring improved use of a system's resources, where requirements could be otherwise considerable if software instance was duplicated for every client. W3C defines a Web 
service as "a software system designed to support interoperable machine-to-machine interaction over a network" $[8,9]$. This definition includes varied systems, but usually refers to clients/servers communicating over web used HTTP protocol. Web services standardize intra interface applications enabling easy access to server applications over networks for clients.

From an end-user's point of view, cloud computing industry usually refers to different delivery models which refer to various cloud computing architecture layers. A commonly heard term is Software as a Service (SaaS) where an application runs on the cloud, eliminating installing and running it on a client computer. Examples of SaaS are Netsuite or Google Apps to personal applications like Gmail, Facebook, or Twitter. A Platform as a Service, (PaaS) facilitates applications development/deployment without the cost/complexity of buying and managing underlying hardware/software layers. Examples include Microsoft's Azure Services Platform, GoogleApp Engine and Amazon's Relational Database Services. The third cloud computing model is Infrastructure as a Service (IaaS) where storage and computing abilities are accessible as a service. Rackspace Cloud Servers, Amazon's S3 storage service, Joyent and Terremark are some IaaS examples.

Traditional and emerging Cloud-based applications include web hosting, social networking and real time data processing each having a different composition, configuration, and deployment requirements. Quantifying performance of scheduling and allocation policy on Cloud infrastructures (software/hardware/services) for different application under varying load, energy performance and system size is a challenging issue today.

In this paper, performance analysis of cloud under different Virtual Machine (VM) capacity is investigated. The VM parameters like RAM and number of processors is varied and resource allocation, CloudSim debt and percentage of VM used are studied. The rest of the paper is organized as follows: an overview of some related works available in the literature is presented in section 2. The materials and methods used for the performance analysis of cloud computing in this paper are detailed in section 3. Results of the simulation are discussed in section 4 and section 5 concludes the paper.

\section{RELATED WORKS}

Though the IT industry gets many cloud computing opportunities, it has to deal with many problems as it is still in the initial stage. Qi Zhang et al., [10] survey cloud computing emphasizing basic concepts, state-of-the-art applications and architectural designs with research challenges. The goal is to ensure a proper understanding of the challenges faced in cloud computing, recognizing significant research areas in this field.

Ostermann et al., [11] analyzed the effectiveness and applicability of recent cloud computing services in scientific computing with Amazon EC2 platform using microbenchmarks and kernels and evaluated clouds services performance. Due to modifications in clouds, results suggest that advanced performance for applicability in the scientific community is required more in the recent cloud services.

Buyyaa et al., [12] defined Cloud computing and provided architecture to form Clouds leveraging Virtual Machines (VMs) technologies using market-oriented resource allocation. To maintain Service Level Agreement (SLA)oriented resource allocation, insights on market-based resource management approaches including both computational risk management and customer-driven service management were provided. Also, previous opinions regarding interconnecting Clouds to form dynamically global Cloud exchanges and markets were illustrated. Next, some representative Cloud platforms developed in industries were introduced, in addition to the current work to realize marketoriented resource Clouds allocation similar to that realized by Aneka enterprise Cloud technology. This work highlights differences between Internet-based services workload and High Performance Computing (HPC) workload. A metanegotiation infrastructure is described to accomplish global Cloud exchanges and markets, and to demonstrate a study regarding harnessing 'Storage Clouds' to increase both performance content delivery.

\section{METHODOLOGY}

CloudSim: is an extensible simulation framework enables smooth modelling of cloud, run simulations, and experiment with ease to analyse Cloud computing infrastructures and application services. CloudSim helps researchers and industry-based developers focus on specific system design issues requiring investigation without getting caught up in low level Cloud-based infrastructures and services [4].

CloudSim includes novel features like: (i) large scale Cloud computing infrastructure modelling and simulation support including data centers on one physical computing node; and (ii) self-contained platform to model data centers, brokers, schedulers and allocating policies. CloudSim's features include: (i) availability of virtualization engine helping to create and manage multiple, independent, and co-hosted virtualized services; and (ii) Ability to switch between space and time shared processing cores allocation to virtualized services. These CloudSim features speed up new algorithms, methods, and protocols development in Cloud computing contributing to paradigm evolution.

A Datacenter manages many hosts which in turn manage VMs in their life cycles. Host is a CloudSim component representing a physical computing server in a Cloud allotted a pre-configured processing capability, storage, memory, and a provisioning policy to allocate processing cores for virtual machines. The Host component implement interfaces supporting modelling and simulation of both single-core and multi-core nodes.

VM allocation [13] creates VM instances on hosts matching essential characteristics such as memory, configurations including software environment, and requirements of a SaaS provider. VM characteristics are memory, processor, storage, and VM scheduling policy. Multiple VM can run on single hosts simultaneously, maintaining processor sharing policies.

Cloudlet is an application component delivering data in a cloud service model and so Cloudlet length, and output file sizes parameter must be greater than or equal to 1 . It also contains various ids for data transfer and application hosting.

\section{RESULTS}

Cloudsim is used for simulating the various scenarios to study the performance of the cloud computing. Performance analysis of cloud under different Virtual Machine (VM) capacity is investigated by varying the VM parameters like RAM and number of processors. The internal memory i.e., RAM of $512 \mathrm{Mb}$ and $1024 \mathrm{Mb}$ and the number of CPUs are varied from one to three. The internal MIPS and the bandwidth is maintained constant at 1000 each. Two Datacenters are created and 20 VM. Total number of cloudlets 
used is 40. The simulations are conducted for four different combinations of RAM and CPU as follows:

\section{1. $512 \mathrm{Mb}$ RAM, $1 \mathrm{CPU}$ \\ 2. $512 \mathrm{Mb}$ RAM, 2 CPU \\ 3. $512 \mathrm{Mb}$ RAM, 3 CPU \\ 4. 1024 Mb RAM, 1 CPU}

Figure 2 shows the time taken for the 40 cloudlets to be executed in different scenarios. Table 1 and Figure 3 show the number of VMs created and the number of cloudlets executed in each datacentre.

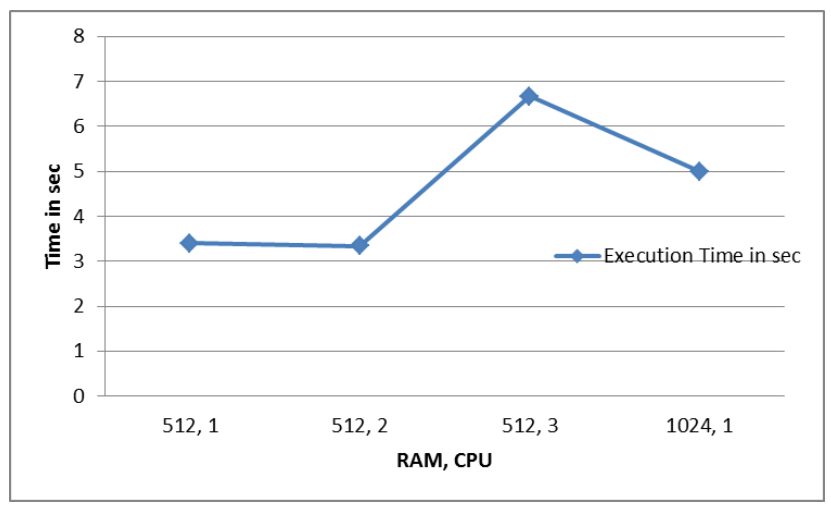

Figure 2: Cloudlets Execution time in sec

Table1: Number of VMs created and the number of cloudlets executed

\begin{tabular}{|l|l|l|l|l|}
\hline & \multicolumn{2}{|l|}{ Datacenter 2 } & Datacenter 3 \\
\hline $\begin{array}{l}\text { RAM, } \\
\text { CPU }\end{array}$ & $\begin{array}{l}\text { Virtual } \\
\text { Machine } \\
\text { created D2 }\end{array}$ & $\begin{array}{l}\text { Cloudlet } \\
\text { executed } \\
\text { D2 }\end{array}$ & $\begin{array}{l}\text { Virtual } \\
\text { Machine } \\
\text { created D3 }\end{array}$ & $\begin{array}{l}\text { Cloudlet } \\
\text { executed } \\
\text { D3 }\end{array}$ \\
\hline 512,1 & 6 & 22 & 6 & 18 \\
\hline 512,2 & 3 & 21 & 3 & 19 \\
\hline 512,3 & 1 & 20 & 1 & 20 \\
\hline $\begin{array}{l}1024, \\
1\end{array}$ & 4 & 20 & 1 & 20 \\
\hline
\end{tabular}

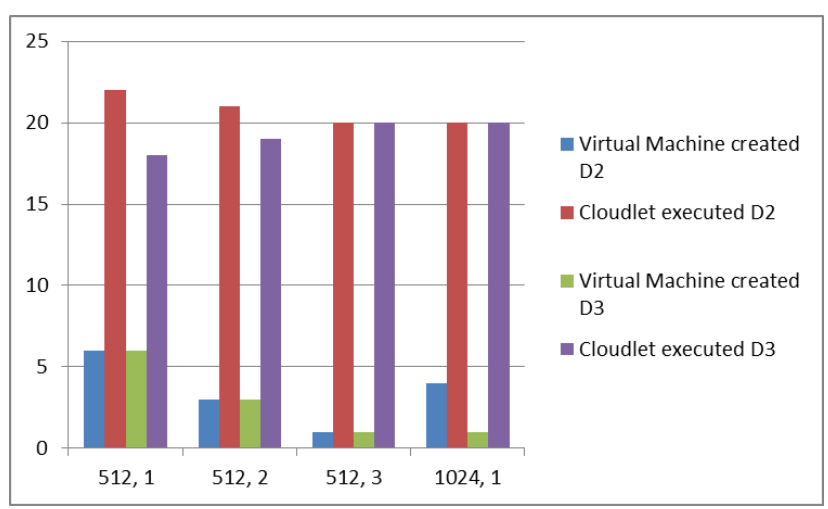

Figure 3: Number of VMs created and the number of cloudlets executed in each datacentre
Debt is calculated according to this formula:

Debt $=$ Ram of $\mathrm{VM}^{*}$ CostPerRam + Size of VM*CostPerStorage

Table 2: Debt incurred for different scenarios

\begin{tabular}{|l|l|}
\hline RAM, CPU & Debt \\
\hline 512,1 & 6153.6 \\
\hline 512,2 & 3076.8 \\
\hline 512,3 & 1025.6 \\
\hline 1024,1 & 4204.8 \\
\hline
\end{tabular}

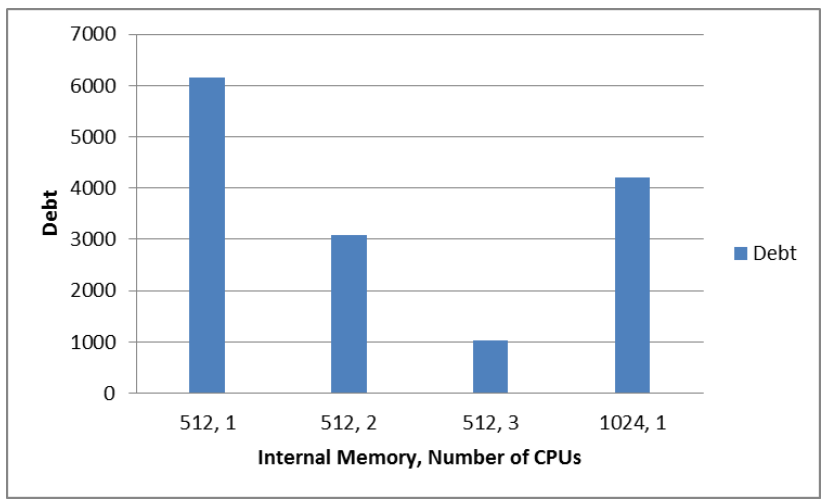

Figure 4: Debt incurred for different scenarios

It is observed from the figures and tables that the varying of the VM characteristics affects the time taken for cloudlet execution, debt incurred. Further investigations are required to study the impact of VMs in cloud computing.

\section{CONCLUSION}

Cloud computing is a business-related infrastructure which is capable of eradicating the importance of high-priced computing hardware and maintenance. In the Cloud computing environment, computing power is supplied by many data centers, installed with hundreds to thousands of servers. Cloudsim simulates various scenarios to study cloud computing performance. Performance analysis of cloud under different Virtual Machine (VM) capacity is investigated by varying the VM parameters like RAM and number of processors. Simulation results demonstrated that the varying of the VM characteristics affects the time taken for cloudlet execution, and debt incurred. Further investigations are required to study the impact of VMs in cloud computing.

\section{REFERENCES}

[1] Weiss. Computing in the clouds. NetWorker, 11(4):1625, Dec. 2007.

[2] R. Buyya, C. S. Yeo, and S. Venugopal. Market-oriented cloud computing: Vision, hype, and reality for delivering IT services as computing utilities. In Proceedings of the 10th IEEE International Conference on High Performance Computing and Communications, 2008.

[3] R. Buyya and M. Murshed, GridSim: A Toolkit for the Modeling and Simulation of Distributed Resource Management and Scheduling for Grid Computing, The Journal of Concurrency and Computation: Practice and 
Experience (CCPE), Volume 14, Issue 13-15, Wiley Press, Nov.-Dec., 2002.

[4] Calheiros, R. N., Ranjan, R., De Rose, C. A., \& Buyya, R. (2009). Cloudsim: A novel framework for modeling and simulation of cloud computing infrastructures and services. arXiv preprint arXiv:0903.2525.

[5] J. E. Smith and R. Nair. Virtual Machines: Versatile platforms for systems and processes. Morgan Kauffmann, 2005.

[6] Marston, S., Li, Z., Bandyopadhyay, S., Zhang, J., \& Ghalsasi, A. (2011). Cloud computing-The business perspective. Decision Support Systems, 51(1), 176-189.

[7] M. A. Vouk, Cloud computing - issues, research and implementations, Journal of Computing and Information Technology 16 (4) (2008) 235-246.

[8] W3C, Web Services Glossary [cited 2009 April 3]; Available from: http://www.w3. org/TR/ws-gloss/.

[9] Marston, S., Li, Z., Bandyopadhyay, S., Zhang, J., \& Ghalsasi, A. (2011). Cloud computing-The business perspective. Decision Support Systems, 51(1), 176-189.
[10] S. Ostermann, A. Iosup, N. Yigitbasi, R. Prodan, T. Fahringer, and D. Epema. A Performance Analysis of EC2 Cloud Computing Services for Scientific Computing. In Cloudcomp, 2009.

[11] Buyya, R., Yeo, C. S., Venugopal, S., Broberg, J., \& Brandic, I. (2009). Cloud computing and emerging IT platforms: Vision, hype, and reality for delivering computing as the 5th utility. Future Generation computer systems, 25(6), 599-616.

[12] Zhang, Q., Cheng, L., \& Boutaba, R. (2010). Cloud computing: state-of-the-art and research challenges. Journal of Internet Services and Applications, 1(1), 7-18.

[13] Quiroz, H. Kim, M. Parashar, N. Gnanasambandam, and N. Sharma. Towards Autonomic Workload Provisioning for Enterprise Grids and Clouds. Proceedings of the 10th IEEE/ACM International Conference on Grid Computing (Grid 2009), Banf, Alberta, Canada, October 13-15, 2009, IEEE Computer Society Press. 